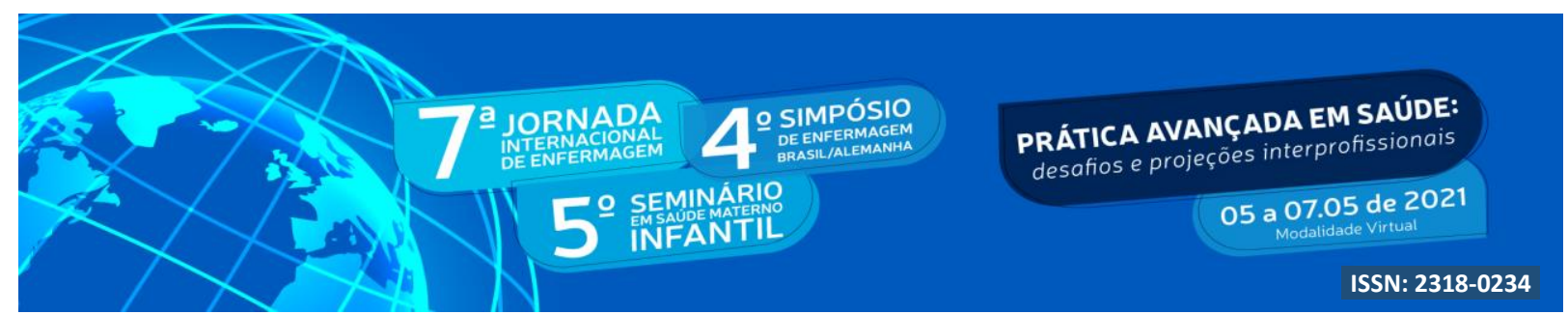

DOI: http://doi.org/10.48195/jie2021-181

\title{
ESTÁGIO CURRICULAR SUPERVISIONADO EM ENFERMAGEM I NA ATENÇÃO PRIMÁRIA À SAÚDE: RELATO DE EXPERIÊNCIA
}

\author{
Francisco Fernandes'; Estevan Guterres de Oliveira²; Marcela Ragagnin 3; Joselaine \\ Silva $^{4}$; Naiana Oliveira dos Santos ${ }^{5}$
}

\begin{abstract}
RESUMO
Relatar o planejamento da ação desenvolvida no Estágio Curricular Supervisionado em Enfermagem I com base nos problemas sociais vivencidos pelo campo prático de determinada comunidade. A presente pesquisa tem como abordagem qualitativa utilizando o diagnóstico situacional do serviço como referência da ação. Dos resultados trata-se de uma ação ilustrativa, bem como os métodos de contracepção e prevenção de infecções sexualmente transmissíveis (IST). Concluiu-se a necessidade de determinada comunidade de conhecimentos na promoção e prevenção da saúde para fim de diminuir os casos de IST e gravidez indesejada.
\end{abstract}

Palavras-chave: Enfermagem; Saúde; Prevenção. Atenção Primária à Saúde

\begin{abstract}
Report the planning of the action developed in the Supervised Curricular Internship in Nursing I based on the social problems experienced by the practical field of a given community. The present research has a qualitative approach using the situational diagnosis of the service as a reference for the action. The results are an illustrative action, as well as methods of contraception and prevention of sexually transmitted infections (STIs). The need for a determined community of knowledge in health promotion and prevention was concluded in order to reduce cases of STIs and unwanted pregnancies.
\end{abstract}

Key Words: Nursing; Health; Prevention; Primary Health Care.

\footnotetext{
${ }^{1}$ Acadêmico de Graduação em Enfermagem - UFN 01franciscofernandes@ gmail.com

2 Acadêmico de Graduação em Enfermagem - UFN estevanguterres@ hotmail.com

3 Preceptora do Estágio Curricular Supervisionado em Enfermagem - UFN marcelaragagnin@gmail.com

${ }^{4}$ Preceptora do Estágio Curricular Supervisionado em Enfermagem - UFN joselainerighe@ hotmail.com

${ }_{5}$ Orientadora. Doutora em Enfermagem. Docente da Universidade Franciscana - UFN naiana.santos@ufn.edu.br 


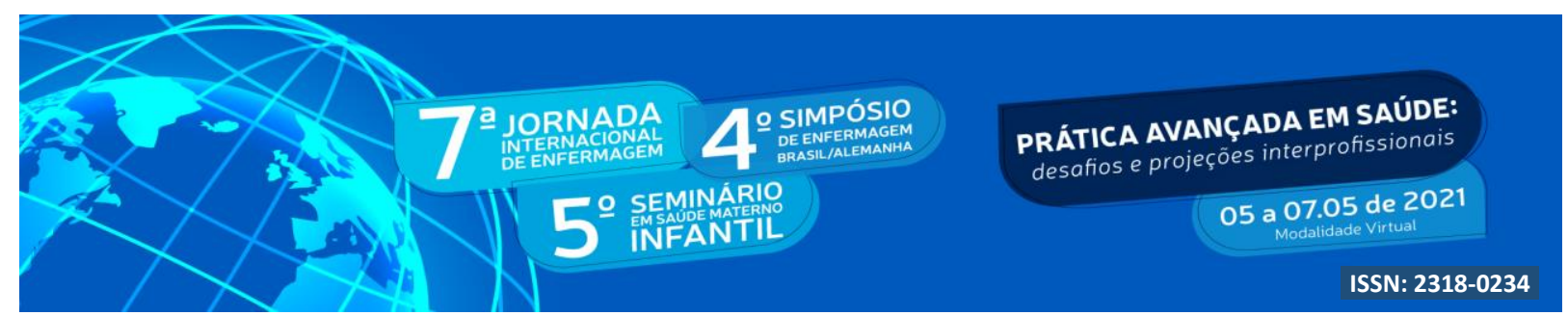

\section{INTRODUÇÃO}

O estágio curricular supervisionado (ECS) é uma ferramenta utilizada para a aproximação dos acadêmicos com os serviços, aperfeiçoando conhecimentos, habilidades e atitudes profissionais, fortalecendo suas competências no processo de trabalho. (ESTEVES; CUNHA; BOHOMOL; NEGRI, 2018).

A atenção primária à saúde (APS) compreende-se com a inserção de unidades básicas baseadas na territorialização do município, para fim de cuidar da saúde de toda a população. Os Territórios são destinados para dinamizar a ação em saúde pública, o estudo social, econômico, epidemiológico, assistencial, cultural e identitário, possibilitando uma ampla visão de cada unidade geográfica e subsidiando a atuação na Atenção Básica, de forma que atendam a necessidade da população adscrita e ou as populações específicas (BRASIL, 2017).

A atenção ao primeiro contato traz em si a ideia de que em um sistema de saúde organizado a partir da APS existe um ponto de entrada (porta de entrada) de fácil acesso para cada novo problema ou novo episódio de um problema pelo qual as pessoas buscam cuidado (PORTELA, 2017).

A demanda de usuários é devido diversas problemas sociais em relação a saúde, porém a problematização que requer maior cuidado e encaminhamentos é a quantidade de casos de Infecções Sexualmete Transmissíveis (IST's) na região e a quantidade de gravidez indesejada pela população jovem/adulto. Os diagnósticos são feitos em dispositivos de testes rápidos para gestação e as infecções mais recorrentes são: Sífilis, HIV, Hepatite B e C, sendo um grande fluxo de atendimento para os profissionais inseridos.

A estrutura física do local possui uma ampla sala de recepção onde os usuários tem acesso a camisinhas como método contraceptivo e barreira a IST's, podendo também para as mulheres pílulas anticoncepcionais de uso mensal ou as de uso trimestral, onde são administradas na sala de procedimentos da UBS. Observou-se com a vivência do serviço a necessidade de um planejamento adequado para a prevenção da saúde dos usuários. Dessa 


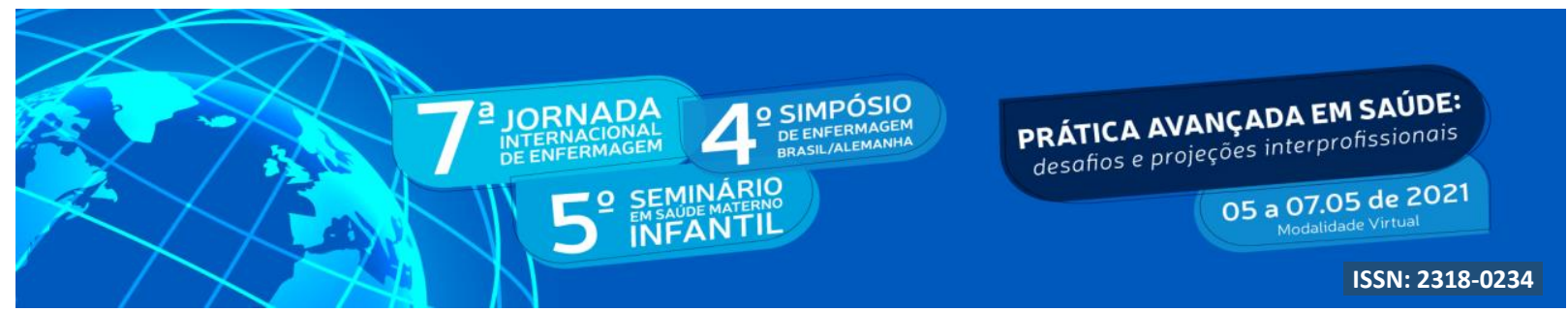

forma, uma estratégia a ser utilizada para combater e controlar as infecções sexualmente transmissíveis é a implantação de dispositivos que diagnostiquem a doença o quanto antes, como os testes rápidos para a sífilis e para o HIV, bem como testes para gonorreia e clamídia e mesmo sendo pouco acessíveis podem ser implantados (SILVA; etal, 2021).

A importância do presente relato é pelas demandas recorrentes de casos com doenças infectocontagiosas, para fim de diminui-las e aumentar a procura do serviço pela prevenção e não pelo tratamento. Cabe salientar que a importância da descrição desta ação é pela preocupação que cada unidade básica, responsável por parte das comunidades de determinado município, se posicionem e planejem ações educativas para combater estes tipos de casos.

\section{OBJETIVO}

Relatar o planejamento da ação desenvolvida no Estágio Curricular Supervisionado em Enfermagem I.

\section{METODOLOGIA}

Trata-se de um estudo do tipo relato de experiência, de abordagem qualitativa, cujas vivências teórico-práticas foram realizadas durante o desenvolvimento do planejamento da ação no Estágio Curricular Supervisionado em Enfermagem I da Universidade Franciscana no curso de Enfermagem, onde o campo de estágio foi na Atenção Básica em Saúde (ABS) na UBS Kennedy, sendo realizado no período de 2020/1 com duração de 140 horas. A primeira etapa foi o diagnóstico situacional das reais necessidades do serviço e dos usuários, após a execução do banner e informações que devem ser relacionadas com o real propósito da ação.

A ilustração deverá ter imagens das pílulas anticoncepcionais e das camisinhas masculinas, femininas e para adolescentes fornecidas no serviço quanto ao uso correto destes métodos, também a importância do uso para a prevenção da saúde e propagação de IST's, também para o planejamento familiar.

O público-alvo tem como objetivo atingir os jovens e também os adultos onde a 


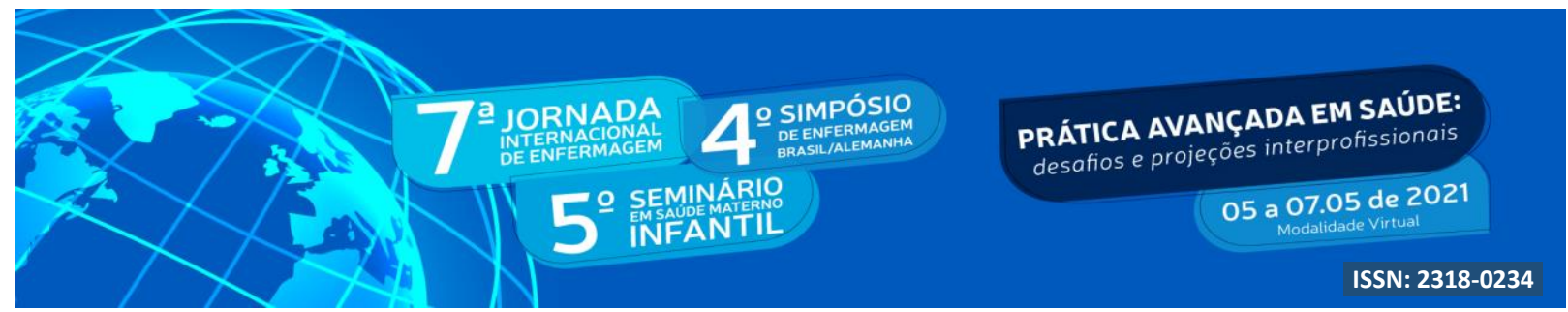

maioria é de classe baixa e pelo grande índice de casos de infecções sexualmente transmissíveis da região em que o serviço está inserido.

\section{DESENVOLVIMENTO}

O diagnóstico situacional do local foi realizado pela vivência adquirida com base nos problemas sociais da unidade básica, sendo notável o alto índice de casos de infecções sexualmente transmissíveis, tais como, sífilis, HIV, hepatite B e HPV.

A necessidade de diminuir os índices de diagnósticos por testes rápidos por conta dos casos de IST's nas comunidades é importante para a prevenção da dissipação de diversas doenças infectocontagiosa. Assim, indagando aos profissionais da saúde inseridos nos diversos serviços em saúde, a organizarem ações que atribuam estratégias para chamar a atenção dos usuários em relação a esta temática.

As IST são importantes causas de procura pelos serviços de saúde e podem provocar sérias complicações, como infertilidade, aborto espontâneo, malformações congênitas, infecções generalizadas e morte, se não tratadas (SOUSA, et al, 2017).

A ação realizada teve relação com o aumento da procura dos usuários nos métodos de prevenção, sendo os preservativos na população adulta e também dos preservativos para adolescentes e melhor conhecimento para ambas as partes. Assim tendo em vista mais encaminhamentos de consulta médica para o inicio do uso de pilulas anticoncepcionais. A promoção da saúde reforça o conceito de determinação social da saúde, com objetivo de impactar favoravelmente a qualidade de vida (HEIDEMANN: WOSNY; BOEHS, 2014).

Ao decorrer das fotografias tiradas para fim de clarear a população dos métodos contraceptivos, e assim passar uma visibilidade das vantagens em que o Sistema Ùnico de Saúde (SUS) disponibilizada a todas comunidades do município, para diferentes tipos de públicos, pode-se visualizar nas respectivas figuras os principais métodos contraceptivos, com o propósito de fazer um informativo aos usuários de determinada comunidade com a aplicação das imagens em um banner e colar na recepção da unidade básica. 


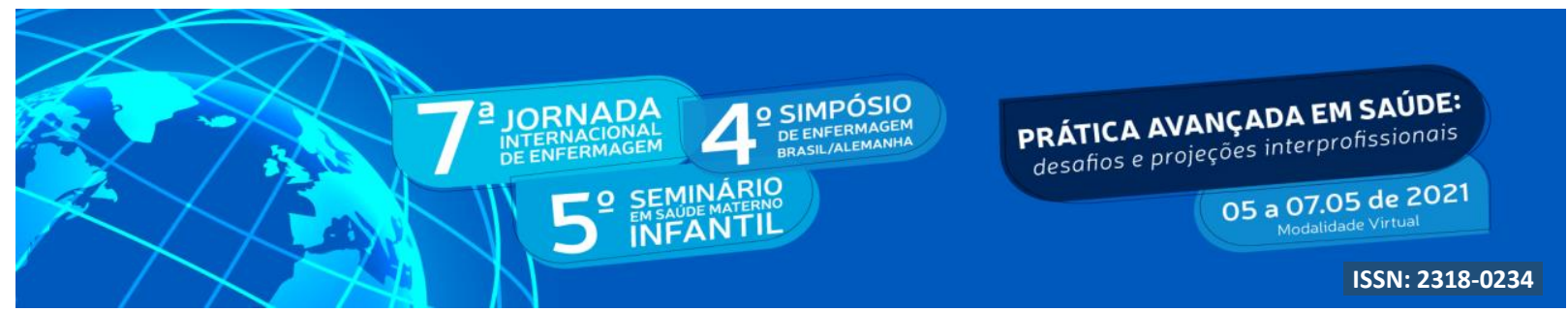

Os métodos contraceptivos disponibilizados gratuitamente são respectivamente: Figura (1) os preservativos masculinos, figura (2) os preservativos para os adolescentes, figura (3) os preservativos femininos, figura (4) os anticoncepcionais administrados mensalmente, figura (5) os anticoncepcionais administrados a cada três mêses.

\subsection{CARACTERÍSTICAS DA UNIDADE}

A Unidade Básica de Saúde (UBS) que foi vivenciada o presente estudo, possui uma grande territorialização, tendo compromisso com diversos bairros onde a quantidade de usuários é relativamente movimentada.

A estrutura do serviço é bem localizada sendo na região norte da cidade de Santa Maria, Rio Grande do Sul, possuindo serviços de Estratégia de Saúde em Família e UBS com atendimentos especificos como curativos de cobertura.

A equipe de saúde apenas da Unidade Básica de Saúde é constituida por 6 médicos, 2 enfermeiras, 4 técnicos de enfermagem e 3 recepcionistas, sendo cada profissional com sua própria carga horária, sendo metade dos médicos com atendimentos especializados como ginecológicos. Os horários de atendimento é das 08:00 ás 12:00 da manhã e por parte da tarde das 13:30 ás 17:00.

O movimento de atendimentos da UBS Kennedy foi exclusivos a casos graves por decorrência da Pandemia do COVID-19 no período da ação, sendo desmarcados todos os procedimentos como preventivos, curativos e solicitação de exames laboratoriais como de mama.

\subsection{Planejamento}

A temática da ação objetivou-se em ilustrar por meio de um banner os métodos contraceptivos que o serviço disponibiliza a comunidade para evitar a gravidez indesejada e a contaminação a IST's. 


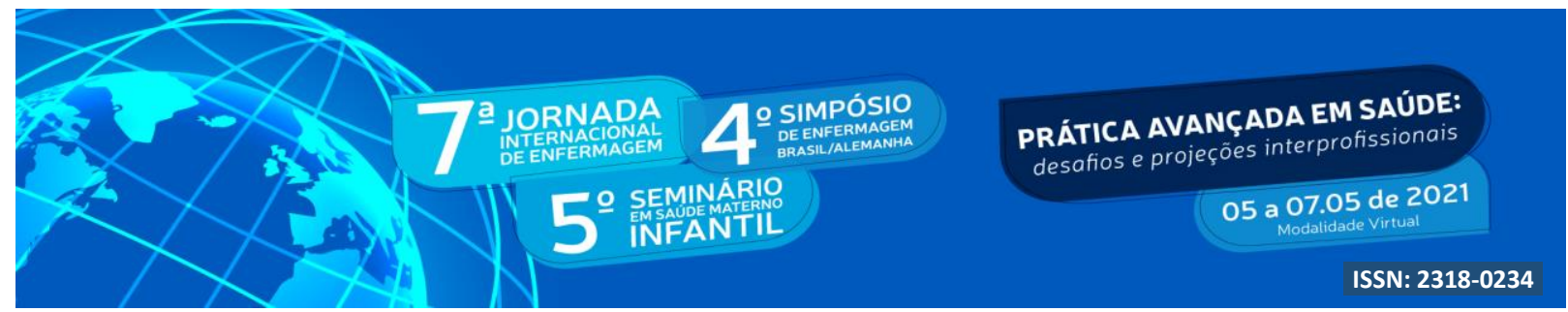

O movimento pela população jovem-adulto na procura de tratamentos é intensa, sendo comumente a administração de benzetacil conforme prescrição médica para tratamentos de casos como sífilis. Encaminhamentos para a unidade de referência municipal a IST's é a casa treze de maio, onde a enfermeira tem contato direto com eles para marcação de consultas presenciais dos pacientes contaminados.

Os jovens inseridos na comunidade parecem não ter conhecimento da importância de se previnir devido a grande quantidade da procura do serviço por sintomas e também por não conhecer os métodos de prevenção a IST's da UBS/ESF, sendo importante que seja explicito na entrada do serviço os dispositivos que são disponibilizados para diminuição da incidência destes casos, sendo gratuito a todos.

A grande demanda da população vai para resolver outros problemas de saúde, porém o fluxo é intenso, e com um banner na recepção próximo ao coletor de camisinhas acredita-se que será de grande relevância, os usuários se interessarão mais em se prevenir, aumentando os atendimentos para prevenção e diminuindo os atendimentos de diagnóstico e tratamento, diminuindo as consultas médicas, procedimentos de administração de medicação, diagnóstico por teste rápido e encaminhamentos para outros serviços.

Figura 1: Preservativo masculino

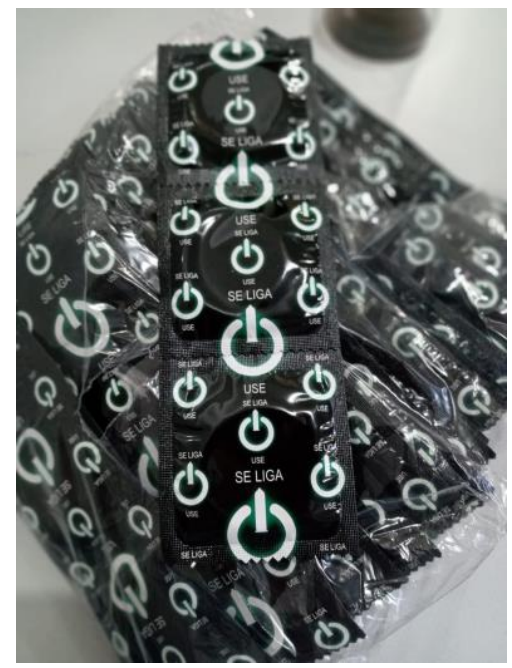




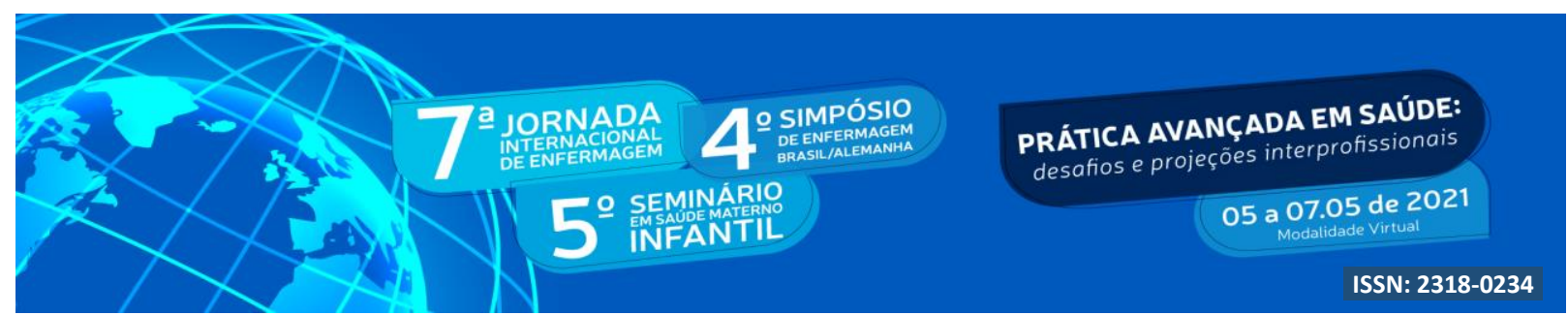

Figura 2: Preservativo para adolescentes

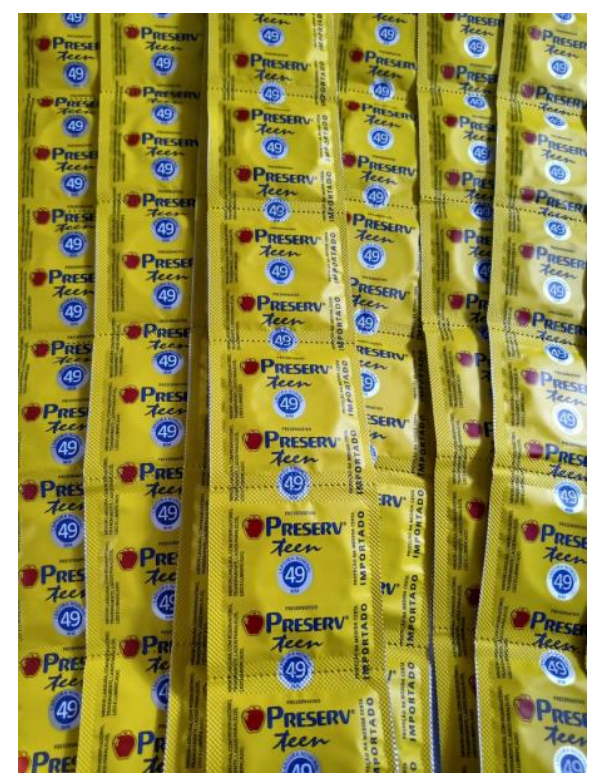

Figura 3: Preservativo feminino

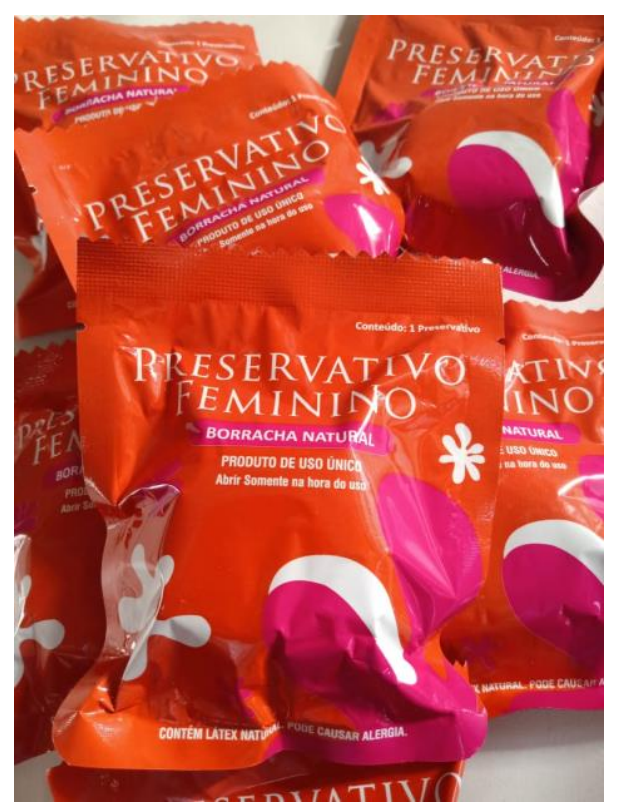




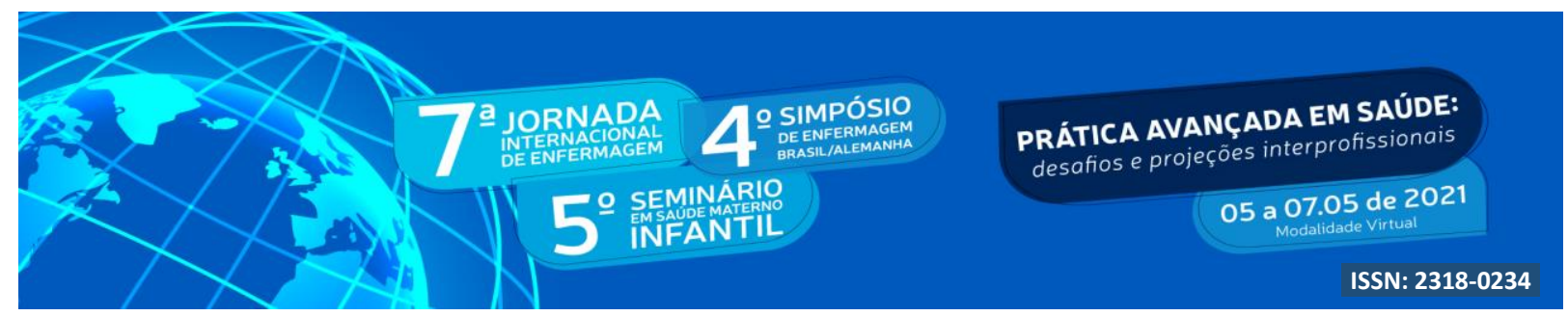

Figura 4: Medimcaneto fornecido conforme prescrição médica, anticoncepcional de uso mensal

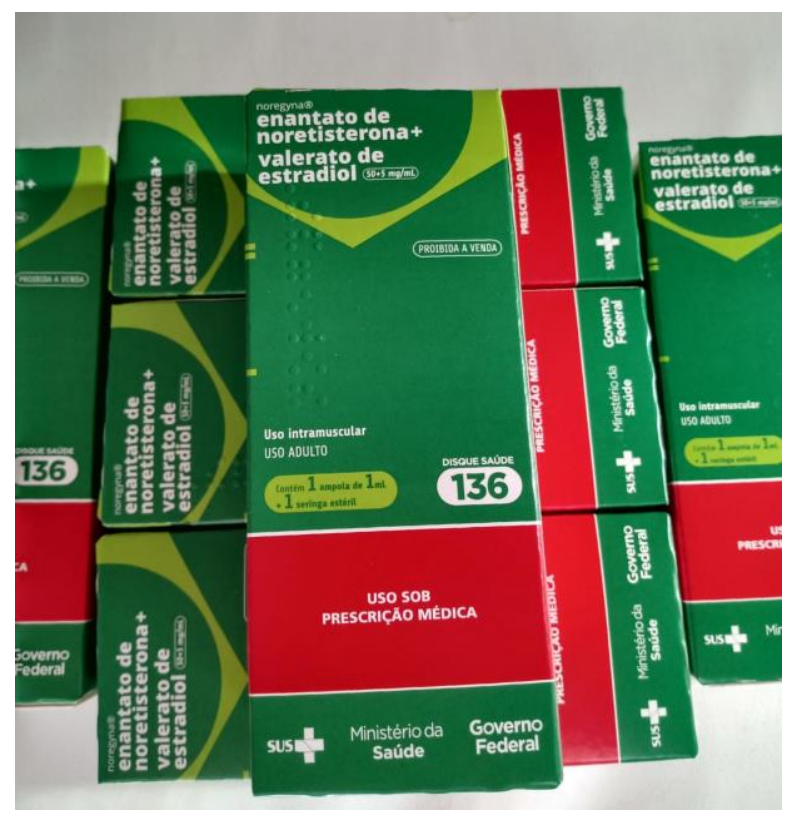

Figura 5: Medicamento fornecido conforme prescrição médica, anticoncepcional de uso trimestral.

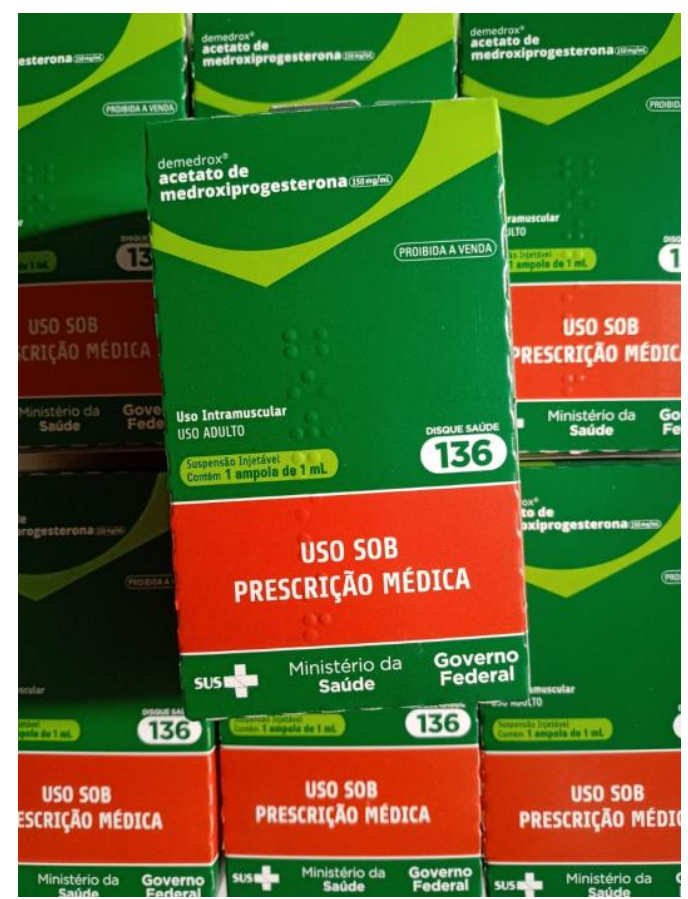




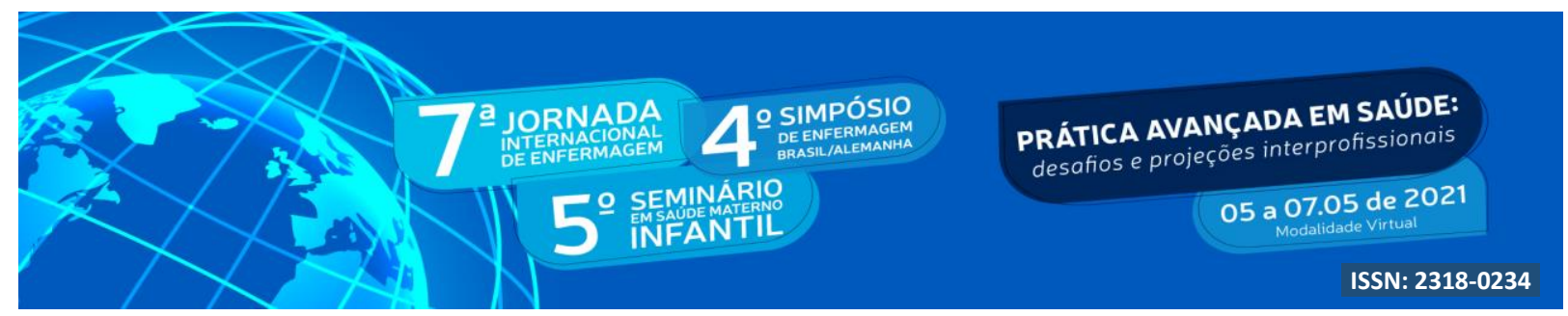

\subsection{Execução}

A elaboração do planjemaneto veio pela necessidade de determinada comunidade, junto com as enfermeiras preceptoras, demais profissionais da unidade e a professora supervisiora do estágio.

O planejamento da ação tem como instrumentos para elaboração imagens anexadas no presente trabalho que ilustram os dispositivos de prevenção, a açao terá inicialmente com um título que descreva os métodos contraceptivos e de prevenção a IST's da UBS Kennedy. Para o designer do banner utilizou-se o windows para a inserção das imagens dos preservativos e anticoncepcionais.

Utilizou-se da ferramenta de planejamneto de ação na atenção primária em saúde para a realização do presente estudo, juntamente com os profissionais inseridos na unidade básica e da Universidade Franciscana. O planejamento é então determinado pela construção de consensos e realiza a combinação de ações estratégicas entre os atores para enfrentar os problemas (TOLEDO, 2008).

\section{CONCLUSÃO}

Concluiu-se com a vivência a visibilidade dos problemas sociais onde o país, estado e município se encontra, tendo como determinante problemas de saúde devido a falta de conhecimento sobre a importância do cuidado. Assim como, mulheres jovens de idade entre quinze a vinte e quatro anos em gestação indesejada. Homens e mulheres com IST's que não se protegiam de nenhuma forma possivel que a UBS fornecia, assim podendo amparar previamente novos casos e também indagar a procura do serviço para o diagnóstico/tratamento dos casos confirmados.

A vivência forneceu um aprendizado pessoal no âmbito do cuidado em enfermagem, pois foi possível notar problemas sociais e também agir sobre eles, como a aplicação de procedimentos com propósito de tratamento e podendo agir com a promoção da saúde e também a prevenção em alguns casos diretamente com o paciente. 


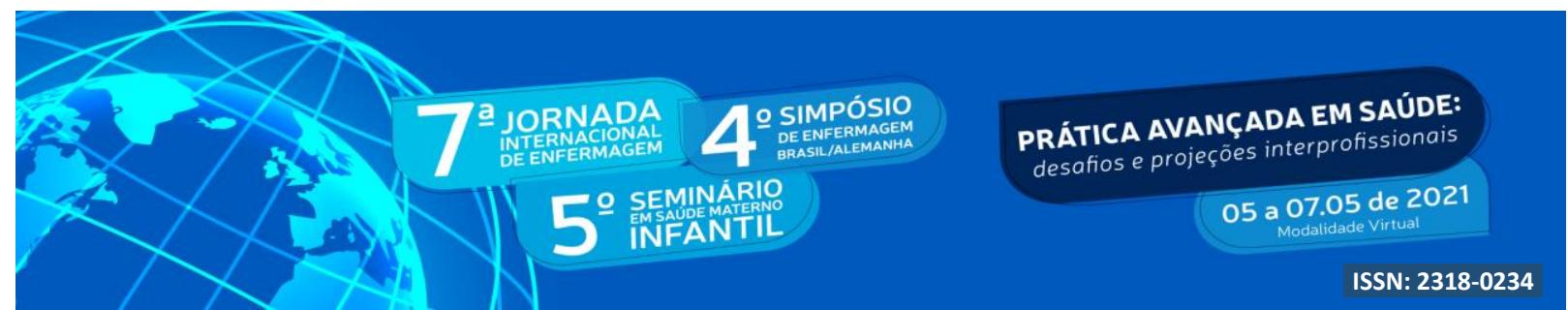

Dessa forma foi possível observar a necessidade das atenções primárias em saúde em promover ações que visam aperfeiçoar o conhecimento da comunidade e em se interessar mais no auto-cuidado.

\section{REFERÊNCIAS}

BRASIL. Ministério da Saúde. POLÍTICA NACIONAL DE ATENÇÃO BÁSICA OPERACIONALIZAÇÃO. Brasília, Cap. 1, 2017. Disponível em: <http://bvsms.saude.gov.br/bvs/saudelegis/gm/2017/MatrizesConsolidacao/comum/250693.ht ml> Acesso em: 18 de abril de 2021.

ESTEVES, L. S. F; CUNHA, I. C. K. O; BOHOMOL, E; NEGRI, E. C. O estágio curricular supervisionado na graduação em enfermagem: revisão integrativa. Rev. Bras. Enferm. 2018; 71:1740-50. DOI: http://dx.doi.org/10.1590/0034-7167-2017-0340.

HEIDEMANN, I. T. S. B; WOSNY, A. M; BOEHS, A. E. Promoção da Saúde na Atenção Básica: estudo baseado no método de Paulo Freire. Departamento de Enfermagem, Centro de Ciências da Saúde, UFSC, 2014, p. 3554. Disponível em: <https://www.scielo.br/pdf/csc/v19n8/1413-8123-csc-19-08-03553.pdf>

PORTELA, G. Z. Atenção Primária à Saúde: um ensaio sobre conceitos aplicados aos estudos nacionais. TEMAS LIVRES • Physis 27 (2) Jan-Mar 2017. Disponível em: <https://www.scielosp.org/article/physis/2017.v27n2/255-276/pt/?abstract_lang=pt\#>

SILVA, P. R. R; ET AL. Estratégias de prevenção a IST realizadas por enfermeiros na atenção primaria a saúde: uma revisão integrativa. Brazilian Journal Of Health review, Vol 4, No 2, 2021

SOUSA, C. P; et al. ADOLESCENTES: MAIOR VULNERABILIDADE ÀS IST/AIDS? RETEP - Rev. Tendên. da Enferm. Profis., 2017; 9(4): 2289-2295. Disponível em: $<$ http://www.coren-ce.org.br/wp-content/uploads/2019/02/ADOLESCENTES-MAIORVULNERABILIDADE-\%C3\%80S-ISTAIDS.pdf>

TOLEDO, P. P. S. Et al. PLANEJAMENTO NA ATENÇÃO PRIMÁRIA À SAÚDE. Guia de Trabalho para o Enfermeiro na Atenção Primária à Saúde, Cap. 7, Pag 135-144, 2008. 